\title{
Seroprevalence of Hepatitis B and C in Patients With Hemophilia: A Single-Centre Descriptive Study
}

\author{
Seyedeh Azra Shamsdin ${ }^{1,2} ;$ Masood Sepehrimanesh $^{1, *} ;$ Babak Pezeshki $^{3} ;$ Maryam Nejabat $^{1,4}$ \\ ${ }^{1}$ Gasteroenterohepathology Research Center, Shiraz University of Medical Sciences, Shiraz, IR Iran \\ ${ }_{2}^{2}$ Prof. Alborzi Clinical Microbiology Research Center, Shiraz University of Medical Sciences, Shiraz, IR Iran \\ 3 Department of Internal Medicine, Fasa University of Medical Sciences, Fasa, IR Iran

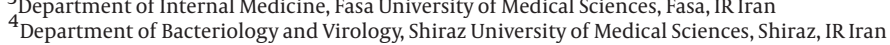 \\ ${ }^{*}$ Corresponding Author: Masood Sepehrimanesh, Gasteroenterohepathology Research Center, Shiraz University of Medical Sciences, P. O. Box: 71935-1311, Shiraz, IR Iran. Tel/Fax: +98- \\ 7116474263, E-mail: sepehrimaneshmasood@gmail.com
}

Received: October 13, 2014; Revised: April 11, 2015; Accepted: June 1, 2015

Keywords: Hepatitis; Hemophilia; Blood Transfer; Coinfection; Prevalence

Hemophilia population is one of the most endangered people, being infected with blood-borne diseases via blood (or its products) transfusion. The aims of this study was to evaluate and compare the seroprevalence of hepatitis B virus (HBV), hepatitis C virus (HCV) and $\mathrm{HBV} / \mathrm{HCV}$ co-infection in hemophilia patients as the population at risk of viral hepatitis infections.

One hundred and forty-six patients with hemophilia who referred to the two main hospitals of Fasa City, Fars Province, Iran during 2007 - 2013 were selected (male to female ratio of approximately 1:1). These patients were checked for HBV and HCV infection using Hepatitis B surface antigen (HBsAg) and anti-HCV antibody (IgG) assay through ELISA method. SPSS software was used for data analyses.

Our sample comprised 57\% men and 43\% women with the mean age of $20.4 \pm 11.9$ years (age range from 0.08 84 years). The prevalence of HBV and HCV infection in patients with hemophilia were $1.4 \%$ (2 patients) and $8.9 \%$ (13 patients), respectively. No patient with HBV/HCV coinfection was detected and also, there were no correlation between gender and having splenectomy with the prevalence of both HBV and HCV infections.

The prevalence of HBV and HCV infections among patients with hemophilia found in this study was in line with approximately all other previous reports from other regions of Iran and this means that both HCV and HBV can be a serious problem in patients with hemophilia. However, further studies in other regions with higher patient number and other detecting methods are highly recommended.

Hemophilia A and B are rare hereditary bleeding disorders, which are caused by mutations in the factor VIII and IX genes (1). Hemophilia could be treated with the whole blood or blood products (2). However, blood transfusion is the main risk factor for transmitting some viruses, such as hepatitis B virus (HBV), hepatitis $C$ virus (HCV), and human immunodeficiency virus (HIV) (3). Hepatitis B, caused by HBV, is the most prevalent cause of human liver disease (4), while, hepatitis $\mathrm{C}$, caused by $\mathrm{HCV}$, is one of the most common chronic blood-borne infections (5). The aims of this study were to determine and compare the prevalence of $\mathrm{HBV}, \mathrm{HCV}$ infections and HBV/HCV coinfection in patients with hemophilia living in Fasa, southern part of Iran.

In this cross sectional single-centre descriptive study, 146 patients diagnosed with hemophilia A, were enrolled. They were treated and registered from 2007 - 2012 and referred to two main hospitals of Fasa. This study was approved by ethics committee of the Fasa university of medical sciences and written informed consent was obtained from each patient. Fasting blood samples were taken into sterile vacutainers without anticoagulant and after centrifugation $(2000 \mathrm{~g}$, $10 \mathrm{~min}$ utes), their serums were obtained. HBsAg and immunoglobulin G (IgG) antibodies against HCV were detected using Enzyme-linked immunosorbent assay (ELISA) (Dia. Pro, Diagnostic Bioprobes Srl, Italy).

All statistical analyses were carried out using SPSS software, version 16.0 (SPSS Inc., Chicago, IL, USA) for Windows. One-sample Kolmogorov-Smirnov test was used to test for normality of distribution of the collected data. All data were expressed as frequency (percentage) and age for all evaluated parameters were expressed as mean \pm standard error of mean (SEM). Correlation analysis was performed to evaluate the relationship between sex and having splenectomy with prevalence of both HBV and HCV infections. P $<0.05$ was considered as significant difference.

The prevalence of HBV and HCV infections in hemo- 
philiacs were $1.4 \%$ and $8.9 \%$, respectively. No patients with or without HBV and HCV infections had coinfection with HIV. Also, no HBV/HCV coinfection was detected in patients with hemophilia A.

The correlation between either gender or splenectomy and HBV or HCV infection were shown and compared in Figure 1. There was no significant correlation between gender or splenectomy and HBV or HCV infection ( $\mathrm{P}>$ 0.05). Our patients were categorized into two age groups, lower and higher than 20 years. The correlation analysis between age and $\mathrm{HBV} / \mathrm{HCV}$ infections demonstrated that there were no significant differences in the prevalence of either HBV or HCV infections with respect to lower or higher than 20 years age groups $(\mathrm{P}=0.860$ and $\mathrm{P}=0.178$ for HBV and HCV infections, respectively).

This is the first cross sectional study in Fasa, south of Iran conducted on the prevalence of HBV and HCV infection in hemophiliacs. These patients are potential carriers of HBV and HCV and can infect other people via several routes, so conducting epidemiological studies on these types of patients has high value and importance. Because of the relatively high prevalence of HBV in Iranian population, this virus remains a major challenge for blood transfusion in
Iranian Blood Transfusion Organization (IBTO) and according to the world health organization classifications, Iran with a prevalence rate of around 3.0\% (2.5\% - 3.5\%) HBs-Ag seropositivity in its population is classified as intermediate for HBV infection (6). In a recent study, the rate of HCV seropositivity for rural population of Kavar city in Fars Province was reported as $0.24 \%$ (7). Also, prevalence of HCV in blood donors in different countries of the developed world has been reported to be between $0.3 \%$ and $1.5 \%$ (8). However, the prevalence of HCV infection in patients with hemophilia in Iran ranged between $29 \%$ - $83.3 \%$ with the lowest rate reported in Zahedan and the highest in Tehran $(9,10)$. In Iran, the prevalence of HBV and HCV infection in the hemophiliacs ranges from $0.7 \%-27 \%$ and $15 \%-80 \%$, respectively (11). In 2012, Assarehzadegan et al. in their study reported the prevalence of $1.1 \%$ for HBs-Ag and $54 \%$ for HCV in Ahvaz, southern part of Iran (11). Our finding (prevalence) is consistent with all reported prevalences, which shows the similar prevalence of this disease in Fasa, southern part of Iran with regard to other regions of Iran. However, we did not perform polymerase chain reaction and had no history of antiviral treatment, which means that there might be an overestimation of HCV prevalence.

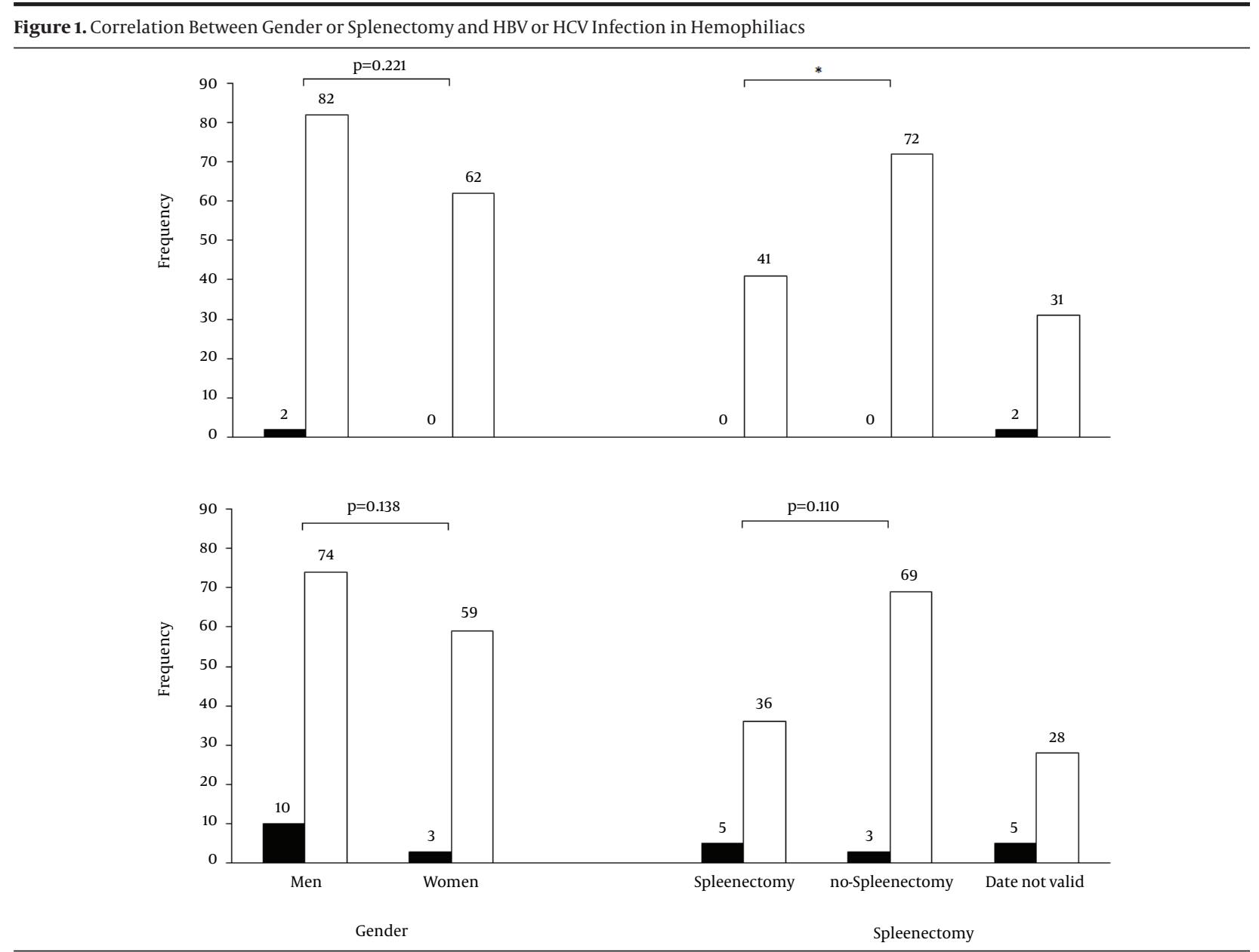

*indicates that no statistics are computed because HBS is a constant. 


\section{Acknowledgements}

Authors wish to thank all staff of gastroenterohepatology research center, Shiraz University of Medical Sciences for their kind assistance in performing this study.

\section{Authors' Contributions}

Study concept and design: Seyedeh Azra Shamsdin. Acquisition of data and drafting of the manuscript: Seyedeh Azra Shamsdin, Masood Sepehrimanesh, Babak Pezeshki and Maryam Nejabat. Statistical analysis and interpretation of data, as well as study supervision: Masood Sepehrimanesh. Critical revision of the manuscript for important intellectual content: Babak Pezeshki.Administrative, technical, and material support: Seyedeh Azra Shamsdin and Maryam Nejabat.

\section{References}

1. Franchini M, Mannucci PM. Past, present and future of hemophilia: a narrative review. Orphanet J Rare Dis. 2012;7:24 .

2. Valizadeh N, Nateghi S, Noroozi M, Hejazi S, Aghanezhad F, Ali AA. Seroprevalence of hepatitis C, hepatitis B and HIV viruses in hemophiliacs born 1985-2010 in west Azarbaijan of Iran. Asian J Transfus Sci. 2013;7(1):55-8.

3. El-Faramawy AA, El-Rashidy OF, Tawfik PH, Hussein GH. Transfu- sion transmitted hepatitis: where do we stand now? A one center study in upper egypt. Hepat Mon. 2012;12(4):286-91.

4. Arababadi MK, Nasiri Ahmadabadi B, Yousefi Daredor H, Kennedy D. Epidemiology of occult hepatitis B infection among thalassemic, hemophilia, and hemodialysis patients. Hepat Mon. 2012;12(5):315-9.

5. Karimi Elizee P, Alavian SM, Miri SM, Behnava B, Alavian SH, Keshvari M, et al. The Seroprevalence of Entrically Transmitted Viral Hepatitis in HCV Infected Thalassemia and Hemophilia Patients in Iran. Jundishapur J Microbiol. 2013;6(7):e9091.

6. Rezvan H, Abolghassemi H, Kafiabad SA. Transfusion-transmitted infections among multitransfused patients in Iran: a review. Transfus Med. 2007;17(6):425-33.

7. Fattahi MR, Safarpour A, Sepehrimanesh M, Hosseini Asl SM, Mohamaddoust $\mathrm{F}$. The prevalence of hepatitis $\mathrm{C}$ virus infection and its related risk factors among the rural population of fars province, southern iran. Hepat Mon. 2015;15(2):e24734.

8. Zanetti AR. Global surveillance and control of hepatitis C. Report of a WHO Consultation organized in collaboration with the Viral Hepatitis Prevention Board, Antwerp, Belgium. $J$ of Viral Hepatitis. 1999;6:35-47.

9. Sharifi-Mood B, Eshghi P, Sanei-Moghaddam E, Hashemi M. Hepatitis $B$ and $C$ virus infections in patients with hemophilia in Zahedan, southeast Iran. Saudi Med J. 2007;28(10):1516-9.

10. Zahedi MJ, Darviesh Moghaddam S. Assessment of prevalence of hepatitis B and C in hemophilic patients in Kerman in 1383. J Kerman Univ Med Sci. 2008;3:131-5.

11. Assarehzadegan MA, Ghafourian Boroujerdnia M, Zandian K. Prevalence of hepatitis B and C infections and HCV genotypes among haemophilia patients in ahvaz, southwest iran. Iran Red Crescent Med J. 2012;14(8):470-4. 